Tema: Metalúrgia Física e comportamento de materiais em temperaturas elevadas

\title{
EFEITO DO TEOR DE SILÍCIO NAS PERDAS HISTERÉTICAS DOS AÇOS DE GRÃO NÃO ORIENTADO*
}

\author{
Adriano Alex de Almeida ${ }^{1}$ \\ Ramon Valls Martins ${ }^{2}$ \\ Fernando Jose Gomes Landgraf ${ }^{3}$
}

\section{Resumo}

Dois aços de grão não orientado com 2,45 e 3,3 \%Si com 7 amostras para cada liga, foram estudados para compreensão do efeito do teor de silício nas perdas histeréticas. As amostras foram recozidas com diferentes temperaturas de recozimento final, resultando em diferentes tamanhos de grão. As perdas histeréticas e $B_{50}$ foram medidos. Os resultados de $B_{50}$ mostraram que não houve variação de textura entre as ligas com diferentes \%Si. Por fim, os resultados não mostraram variações da energia dissipada pela perda histerética com adição de \%Si.

Palavras-chave: Aços de grão não orientado; Tamanho de grão; Perdas histeréticas; Teor de silício.

\section{EFFECT OF THE SILICON CONTENT IN HYSTERESIS LOSS OF NON- ORIENTED STEELS}

\section{Abstract}

Two non-oriented steels with 2.45 and $3.3 \%$ Si with 7 samples for each alloy and same thickness were studied to understand the effect of the silicon content in the hysteresis losses. The samples were annealed with different final annealing temperatures getting different grain sizes. Hysteresis loss and B50 were measured. The results of B50 showed no variation in texture between the alloys with different \%SI. Finally, the results show no variation of the energy dissipated by hysteresis loss with variation of \% $\mathrm{Si}$

Keywords: Non-oriented steels; Silicon content; Hysteresis loss.

1 Engenheiro de Materiais, Mestre em Engenharia Metalúrgica e Materiais, Doutorando em Engenharia Met. e Materiais, Universidade de São Paulo, São Paulo, SP, Brasil.

2 Engenheiro Mecânico, Pós-Doutorado em Física IFUSP, Pesquisador do Instituto de Pesquisas Tecnológicas, São Paulo, SP, Brasil.

3 Engenheiro Metalúrgico, Doutor em engenharia Metalúrgica e Materiais, Diretor Presidente do Instituto de Pesquisas Tecnológicas (IPT), Professor do Departamento de Engenharia Metalúrgica e de Materiais, Escola Politécnica, USP, São Paulo, SP, Brasil.

\footnotetext{
* Contribuição técnica ao $69^{\circ}$ Congresso Anual da ABM - Internacional e ao 14ํㅡㄹ ENEMET - Encontro Nacional de Estudantes de Engenharia Metalúrgica, de Materiais e de Minas, 21 a 25 de julho de 2014, São Paulo, SP, Brasil.
} 


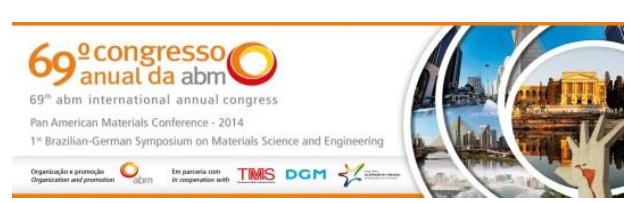

\section{INTRODUÇÃO}

A influência da adição de elementos de liga nos aços GNO tem sido investigada por pesquisadores, como mostra as referências $[1,2]$. Contudo nenhum outro elemento tem tanta importância como o silício. O Si é responsável pela redução das perdas $\left(\mathrm{W} / \mathrm{m}^{3}\right)$ de excesso $\left(\mathrm{P}_{\mathrm{e}}\right)$, e principalmente das perdas parasitas $\left(\mathrm{P}_{\mathrm{p}}\right)$, pelo aumento da resistividade elétrica $(\rho)$.

$\mathrm{O}$ efeito do teor de Si nas perdas de excesso foi mostrado por Bertotti [3] e Campos [4]. As perdas devido às correntes parasitas são inversamente proporcionais à resistividade elétrica (\%Si), vide Thompson [5]. Entretanto nas perdas histeréticas $\left(P_{h}\right)$ o Si não tem influência primária.

Outras propriedades magnéticas são afetadas pelo silício. É o caso da energia magnetocristalina, através da constante magnetocristalina de primeira ordem $\mathrm{K}_{1}$, efeito mostrado pela Equação (Equação 1), onde os Si é dado em \% peso [6]. A polarização de saturação, Js, também é reduzida com a adição de Si Equação (Equação 2) [7], Si em \% peso. Outra propriedade intrínseca que vale ser lembrada é temperatura de Currie ,Tc, que é inversamente proporcional ao teor de Si [8].

$$
\begin{array}{r}
\mathrm{K}_{1}=4,8-0,42(\% \mathrm{Si})\left(\mathbf{1 0}^{\mathbf{4}} \frac{\mathrm{J}}{\mathbf{m}^{3}}\right) \\
\mathrm{J}_{\mathrm{S}}=2,16-0,048(\% \mathrm{Si})(\mathrm{T})
\end{array}
$$

A energia dissipada pela perda histerética é aproximadamente proporcional à energia da parede de domínio, $\gamma$, que é proporcional a raiz quadrada de $K_{1}$ [7]. Portanto é esperado que a $P_{h}$ ou energia dissipada pela perda histerética $\left(W_{h}\left(J / m^{3}\right)\right)$ decresça com a adição de silício [9].

O tamanho de grão e a textura são parâmetros importantes, e com forte influência nas perdas histeréticas [4,10]. Um método bastante útil e prático de avaliação indireta da textura [11] é o $B_{50}$, trata-se da indução magnética medida quando se aplica um campo de $5000 \mathrm{~A} / \mathrm{m}$. O tamanho de grão $(\zeta$ e a textura estão diretamente ligados, é sabido que o aumento do tamanho de grão leva a uma piora da textura [12].

\section{MATERIAIS E MÉTODOS}

Foram utilizadas duas ligas, L1 e L2, composição química apresentada na tabela (Tabela 1), no total de 14 amostras de aço GNO com mesma espessura $0,64 \mathrm{~mm}$. As ligas foram tratadas termicamente sob a mesma sequência de temperatura, na Aperam (Timoteo MG), para crescimento de grão. As temperaturas de recozimento final e o número de identificação de cada amostra estão expostos na Tabela (Tabela 2). Os dados de densidade e a resistividade estão na Tabela (tabela 3).

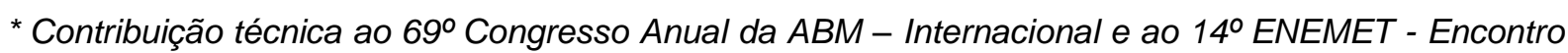
Nacional de Estudantes de Engenharia Metalúrgica, de Materiais e de Minas, 21 a 25 de julho de 2014, São Paulo, SP, Brasil.
} 
Tabela 4. Número das amostras de cada liga e seu respectivo tamanho de grão.

\begin{tabular}{cccc}
\hline $\begin{array}{c}\text { Liga L1 } \\
\text { Amostra }\end{array}$ & $\begin{array}{c}\text { TG } \\
\mu m\end{array}$ & $\begin{array}{c}\text { Liga L2 } \\
\text { Amostra }\end{array}$ & $\begin{array}{c}\text { TG } \\
\mu m\end{array}$ \\
\hline 1 & 53 & 8 & 48 \\
\hline 2 & 68 & 9 & 64 \\
\hline 3 & 100 & 10 & 84 \\
\hline 4 & 121 & 11 & 117 \\
\hline 5 & 152 & 12 & 151 \\
\hline 6 & 176 & 13 & 172 \\
\hline 7 & 249 & 14 & 207 \\
\hline
\end{tabular}

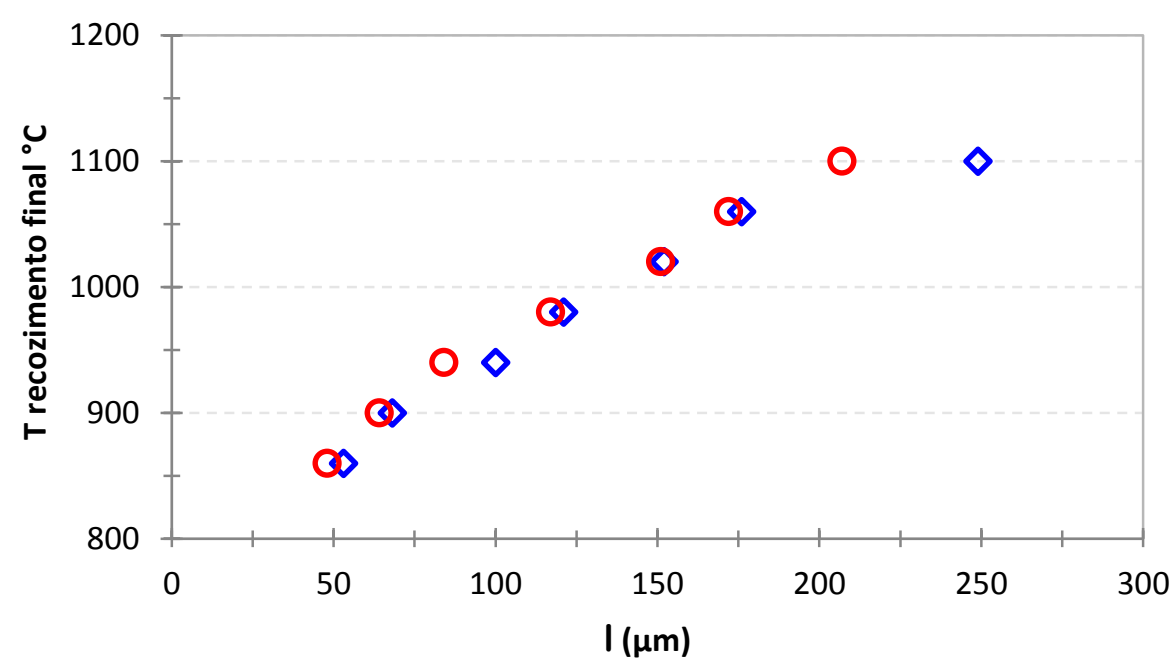

\section{$\diamond$ Liga L1 $\quad$ O Liga L2}

Figura 1. Temperatura de recozimento final por tamanho de grão das amostras da liga 1 e 2 .

* Contribuição técnica ao 69ํㅡㄹ Congresso Anual da ABM - Internacional e ao 14 ENEMET - Encontro Nacional de Estudantes de Engenharia Metalúrgica, de Materiais e de Minas, 21 a 25 de julho de 2014, São Paulo, SP, Brasil. 


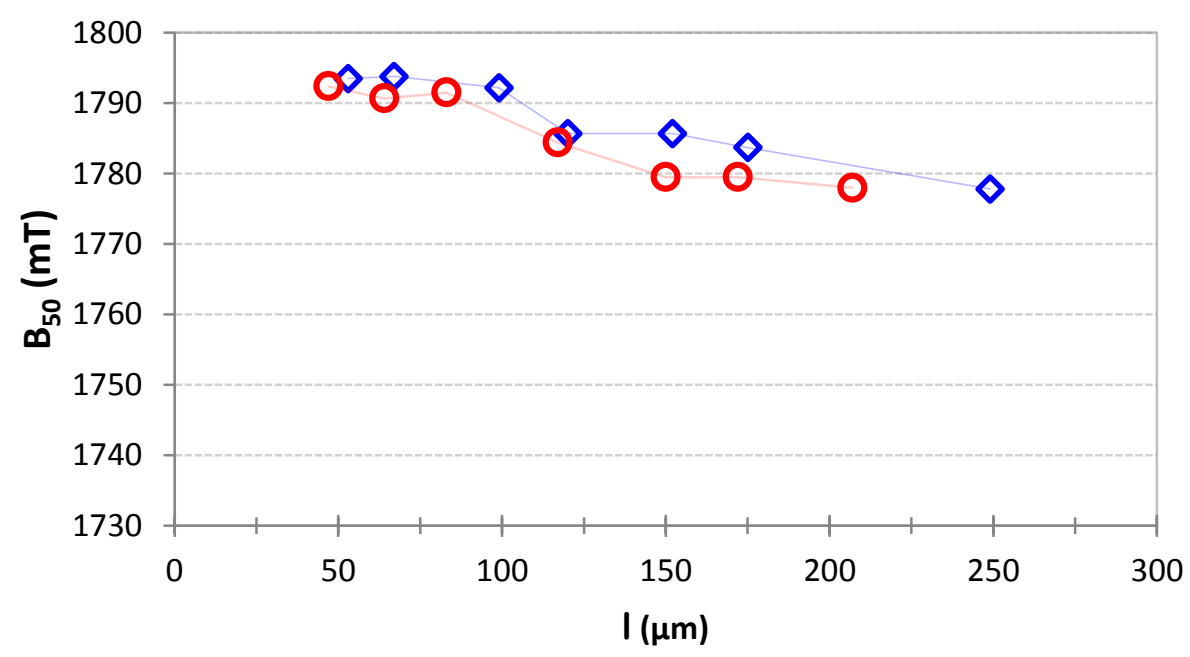

$\diamond$ Liga $1 \quad 0$ Liga 2

Figura 3. B50 com desconte de \%Si em função do tamanho de grão das amostras da liga 1 e 2 .

\subsection{Influência do \%Si nas Perdas Histeréticas}

A fim de investigar o efeito do \%Si, foram comparados dois pares de tamanhos de grãos semelhantes, como consta na Tabela (Tabela 5).

Tabela 5. Pares de tamanho de grão de mesmo tamanho.

\begin{tabular}{cccc}
\hline $\begin{array}{c}\text { Liga L1 } \\
\text { Amostra }\end{array}$ & $\begin{array}{c}\text { TG } \\
\mu \mathrm{m}\end{array}$ & $\begin{array}{c}\text { Liga L2 } \\
\text { Amostra }\end{array}$ & $\begin{array}{c}\text { TG } \\
\mu \mathrm{m}\end{array}$ \\
\hline 2 & 68 & 9 & 64 \\
\hline 5 & 152 & 12 & 151 \\
\hline
\end{tabular}

Na Figura (Figura 4) é mostrada a energia dissipada pela perda histerética $\left(\mathrm{W}_{\mathrm{h}}\right)$ em função da indução magnética, para os tamanhos de grão próximos, Tabela (Tabela 4). Nota-se que não há diferença de $W_{h}$ entre as amostras comparadas, contrariando os resultados de Matsumura [7], que atribui à variação da $W_{h}$ devido à variação de $\mathrm{K}_{1}$. Para a diferença de $\mathrm{Si}$ no presente trabalho $\mathrm{K}_{1}$ teve uma variação de $0,1 \%$ entre as ligas. Os resultados aqui exposto divergem também de Hou [9], o autor mostra uma variação de $W_{h}$ com $\mathrm{Si}$, e que tal variação é mais acentuada para induções de 1,5 T. No gráfico da Figura (Figura 4) a Wh é a mesma tanto para baixas induções quanto para altas induções, tomando a permeabilidade máxima com limite entre alta e baixa indução.

* Contribuição técnica ao 69 Congresso Anual da ABM - Internacional e ao 14ํㅡㄹ ENEMET - Encontro Nacional de Estudantes de Engenharia Metalúrgica, de Materiais e de Minas, 21 a 25 de julho de 2014, São Paulo, SP, Brasil. 


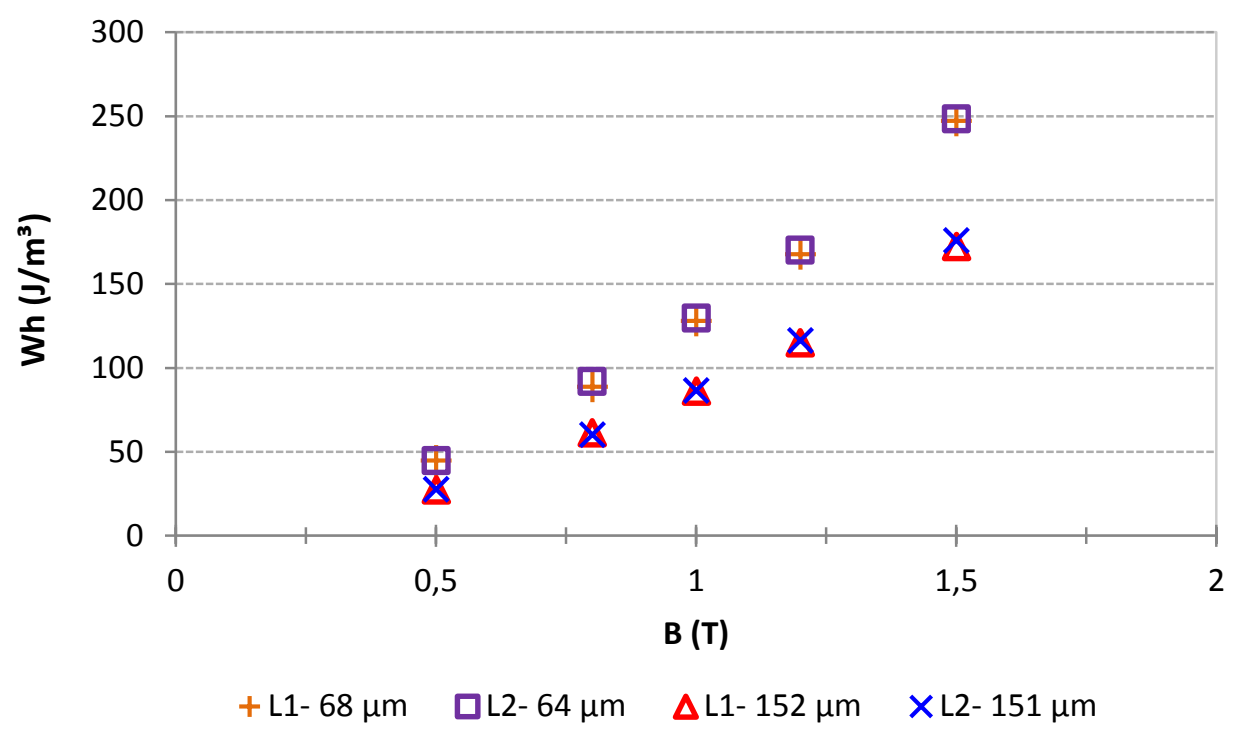

Figura 4. Energia dissipada pela perda histerética em função da B para /próximos.

Uma vez que $J_{s}$ é afetado pela adição de $\mathrm{Si}$, a indução magnética também é. Esse efeito tem consequências na $W_{h}$. Por tanto a energia dissipada na perda histerética é proporcional a polarização magnética efetiva, que é a razão entre a $\mathrm{J}$ do ferro $\left(\mathrm{J}_{\mathrm{Fe}}\right)$ e J do FeSi (Js) [7]. Assim, como a lei de Stteinmetz [14] mostra que a indução máxima afeta $W_{h}$, pode ser proposto uma relação (Equação 4) combinando a polarização efetiva com influência do Si na energia da parede de domínio [7].

\section{CONCLUSÃo}

$$
W_{h} \propto\left(\frac{J_{F e}}{J_{s}}\right)^{1,6} x \sqrt{K_{1}}
$$

A partir dos resultados é dito que:

As perdas histeréticas, ou a energia dissipada pela perda histerética, não variaram com o \% $\mathrm{Si}$, os resultados foram confirmados para diferentes induções magnéticas.

$\mathrm{O}$ tamanho de grão aumentou com a temperatura de recozimento final. $\mathrm{O}$ aumento do tamanho de grão se deu por crescimento de grão.

As ligas não apresentaram diferença de textura, apesar de apresentarem uma piora na textura com o aumento do tamanho de grão.

\section{Agradecimentos}

Os autores agradecem a Aperam South America e ao Instituto de Tecnologia de São Paulo - IPT. À Coordenação de Aperfeiçoamento de Pessoal de Nível Superior (CAPES) pelo apoio financeiro.

* Contribuição técnica ao 69ํㅡㄹ Congresso Anual da ABM - Internacional e ao 14º ENEMET - Encontro Nacional de Estudantes de Engenharia Metalúrgica, de Materiais e de Minas, 21 a 25 de julho de 2014, São Paulo, SP, Brasil. 


\section{REFERÊNCIAS}

1 Rodrigues MF, et al. Texture and magnetic properties improvement of a 3\% Si non-oriented electrical steel by $\mathrm{Sb}$ addition. Journal of Magnetism and Magnetic Materials, 2013. 331(0): 24-27.

2 Wan Y, Chen W, Wu S, Effect of lanthanum content on microstructure and magnetic properties of non-oriented electrical steels. Journal of Rare Earths, 2013; 31(7): 727-733.

3 Bertotti G, General properties of power losses in soft ferromagnetic materials. Magnetics, IEEE Transactions on, 1988; 24(1): 621-630.

4 Campos MF, Teixeira JC, Landgraf FJG. The optimum grain size for minimizing energy losses in iron. Journal of Magnetism and Magnetic Materials, 2006; 301(1): 94-99.

5 Thompson JJ. On the heat produced by Eddy Currents in an iron plate exposed to an alternating magnetic field. The Electrician, 1892; 28.

6 Landgraf F. Nonoriented Electrical Steels. JOM, 2012; 64(7): 764-771.

7 Matsumura K, Fukuda B. Recent developments of non-oriented electrical steel sheets. Magnetics, IEEE Transactions on, 1984; 20(5): 1533-1538.

8 Littmann MF. Iron and silicon-iron alloys. Magnetics, IEEE Transactions on, 1971; 7(1): 48-60.

9 Hou C-K. Effect of silicon on the loss separation and permeability of laminated steels. Journal of Magnetism and Magnetic Materials, 1996; 162(2-3): 280290.

10 Landgraf FJG, et al. Modelling the angular dependence of magnetic properties of a fully processed non-oriented electrical steel. Journal of Magnetism and Magnetic Materials, 2003; 254-255(0): 328-330.

11 Park J-T, Szpunar JA. Effect of initial grain size on texture evolution and magnetic properties in nonoriented electrical steels. Journal of Magnetism and Magnetic Materials, 2009; 321(13): 1928-1932.

12 Cunha MA, Paolinelli SC. Effect of the Annealing Temperature on the Structure and Magnetic Properties of 2\% Si Steel. Materials Research, 2002; 5: p. 373-378.

13 ASTM, Standard Test Methods for Determining Average Grain Size, in ASTM International1996, 2004: West Conshohocken.

14 Steinmetz CP. On the law of hysteresis. Proceedings of the IEEE, 1884; 72(2): 197-221.

* Contribuição técnica ao 69 Congresso Anual da ABM - Internacional e ao 14ํㅡㄹ ENEMET - Encontro Nacional de Estudantes de Engenharia Metalúrgica, de Materiais e de Minas, 21 a 25 de julho de 2014, São Paulo, SP, Brasil. 